\title{
Enhanced-view totally extraperitoneal repair in a patient with incisional hernia after robot-assisted laparoscopic radical prostatectomy: a case report
}

\author{
Shusaku Honma* ${ }^{*}$, Keisuke Tanino, Takashi Kumode, Ryosuke Mizuno, Yugo Matsui, Siyuan Yao, \\ Teppei Murakami, Takatsugu Kan, Sanae Nakajima and Takehisa Harada
}

\begin{abstract}
Background: Although laparoscopic incisional hernia repair, especially laparoscopic intraperitoneal onlay mesh, is a widely used technique, it can cause serious complications, including mesh erosion, adhesive bowel obstruction, and chronic pain. The enhanced-view totally extraperitoneal (eTEP) technique has been reported to prevent such complications by placing the mesh in the retrorectus space. Here, we report the case of a patient with post-robot-assisted laparoscopic radical prostatectomy (RARP) incisional hernia repaired using the eTEP technique.

Case presentation: A 67-year-old man, who underwent RARP for prostate cancer 4 years ago developed an incisional hernia. Abdominal computed tomography showed the presence of an epigastric incisional hernia measuring $4 \mathrm{~cm}$ long and $3.7 \mathrm{~cm}$ wide. We performed an eTEP repair. We closed the hernia defect using a 0 barbed suture and placed a self-gripping mesh measuring $20 \mathrm{~cm}$ long and $15 \mathrm{~cm}$ wide in the developed retrorectus space with no fixation. There were no postoperative complications, and the patient was discharged on postoperative day 2.
\end{abstract}

Conclusions: ETEP repair is considered an extremely effective surgical treatment option for incisional hernias because of its few resulting postoperative mesh-and-tacker-related complications.

Keywords: Incisional hernia, eTEP, RARP, Ventral hernia

\section{Background (Introduction)}

Recently, robot-assisted procedures have become widely used in various surgeries; in particular, robot-assisted laparoscopic radical prostatectomy (RARP) for prostate cancer has been one of the most accepted surgeries. RARP has clinical, functional, and oncological benefits in patients with prostate cancer $[1,2]$. However, according to recent studies, the incidence rate of incisional hernia is higher with minimally invasive radical prostatectomy,

*Correspondence: shomma74@kuhp.kyoto-u.ac.jp Department of Surgery, Kobe City Medical Center West Hospital, 2-4, Ichibancho, Nagataku, Kobe, Hyogo 653-0013, Japan including RARP, than with traditional open radical prostatectomy $[3,4]$.

Incisional hernia is one of the most common postoperative complications experienced by patients, which not only causes pain and cosmetic problems, but also causes incarceration of the small bowel [5]. Emergency surgery is necessary for the incisional hernia incarceration. Even without incarceration, surgical treatment is required to cure the incisional hernia, often leading to further physical and economic burdens on the patient [5].

The enhanced-view totally extraperitoneal (eTEP) technique, an endoscopically performed Rives-Stoppa technique, was first described by Miserez for ventral hernia repair [6]. The eTEP technique has been reported to be 
able to avoid common mesh-and-tacker-related complications from laparoscopic intraperitoneal onlay mesh (IPOM) technique, i.e., mesh erosion, adhesive bowel obstruction, and chronic pain, by placing the mesh in the retrorectus space [7].

To the best of our knowledge, no other study has reported a case of eTEP repair in a patient with incisional hernia after RARP. Here, we report a case of a patient with post-RARP incisional hernia repaired using the eTEP technique.

\section{Case presentation}

A 67-year-old man presented to our department with a swelling in the upper abdomen for half a year. His surgical history included RARP for prostate cancer in 2017. He had been medicated with a few anti-anxiety drugs for depressive anxiety disorder. Physical examination revealed a tennis ball-sized irreducible subcutaneous mass on the previous RARP incision scar, where the specimen was removed from the upper abdominal midline. Blood tests, including blood cell counts, liver enzymes, renal function, and $\mathrm{HbA1c}$, were within the respective reference ranges. Abdominal computed tomography showed the presence of an epigastric incisional hernia $4 \mathrm{~cm}$ long and $3.7 \mathrm{~cm}$ wide (Fig. 1). We decided to perform an eTEP repair.

The patient was placed in the supine position with his arms tucked at the sides under general anesthesia. Figure 2 shows the port placement in this case. We made a $2-\mathrm{cm}$ skin incision at the port 1 position and identified the anterior rectus sheath, then incised it sharply. We used balloon dissector to develop the left retrorectus space after splitting the rectus abdominis muscle,

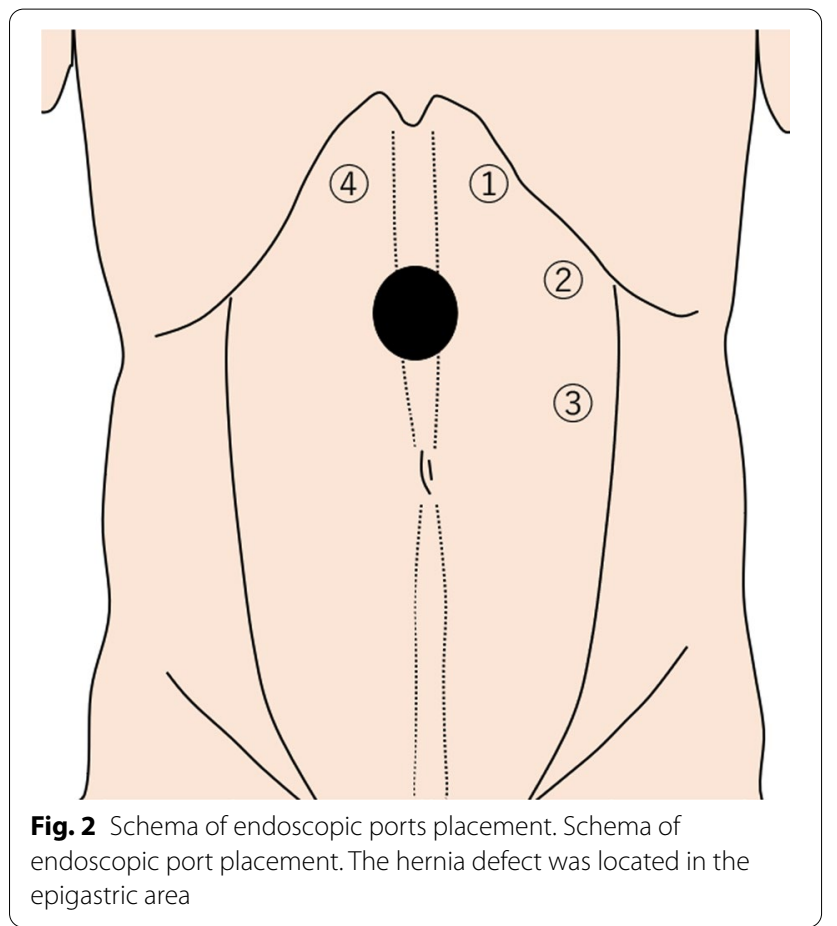

inserted a 12-mm trocar, and performed pneumoperitoneum at $10 \mathrm{mmHg}$. Two $5-\mathrm{mm}$ trocars were inserted at port 2 and port 3 positions under the endoscopic view.

Above the level of the hernia defect, the medial portion of the left posterior rectus sheath was incised from the cephalad in the caudal direction and crossing to the preperitoneal space under the white line to the right posterior rectus sheath. The medial portion of the right posterior rectus sheath was incised and a 12-mm trocar was
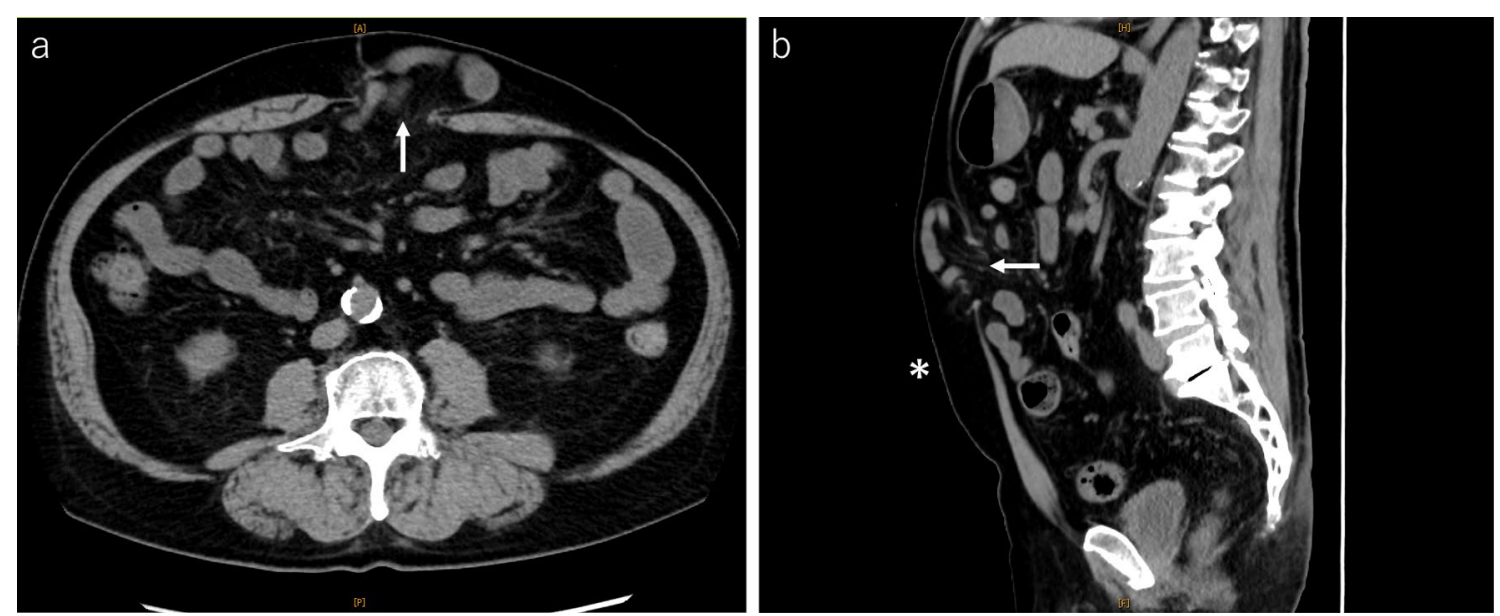

Fig. 1 Abdominal computed tomography. Abdominal computed tomography demonstrates an incisional hernia orifice in the upper abdomen. a Axial image showing the hernia defect measuring $3.7 \mathrm{~cm}$ wide (arrow). b Sagittal image showing the hernia defect measuring $4.0 \mathrm{~cm}$ long (arrow). The defect is located in the upper umbilicus $\left(^{*}\right)$ 
inserted at the port 4 position after sufficient dissection in the right retrorectus space. Retrorectus dissection was performed in the caudal direction, incising bilateral posterior rectus sheaths to the hernia defect (Fig. 3).

When the hernia sac was sharply dissected, we found very mild adhesions between the hernia sac and omental tissue (Fig. 4). Careful adhesiolysis was performed while confirming that there were no adhesions between the hernia sac and intestine. Bilateral retrorectus dissection was performed caudal to the arcuate line. After development of sufficient preperitoneal and bilateral retrorectus spaces, abdominal defect was closed by 0 non-absorbable barbed suture, and defect of the peritoneum was closed by $2-0$ absorbable suture. Finally, a $20-\mathrm{cm}$-long and 15 -cm-wide self-gripping mesh was placed in the retrorectus space with no fixation (Fig. 5). The operating time was $211 \mathrm{~min}$, and the amount of bleeding was $5 \mathrm{~g}$.

There were no postoperative complications and the patient was discharged on postoperative day 2. Postoperative pain was well controlled with prophylactic oral analgesics for 5 days after surgery. No hernia recurrences occurred after 9 months of follow-up (Fig. 6).
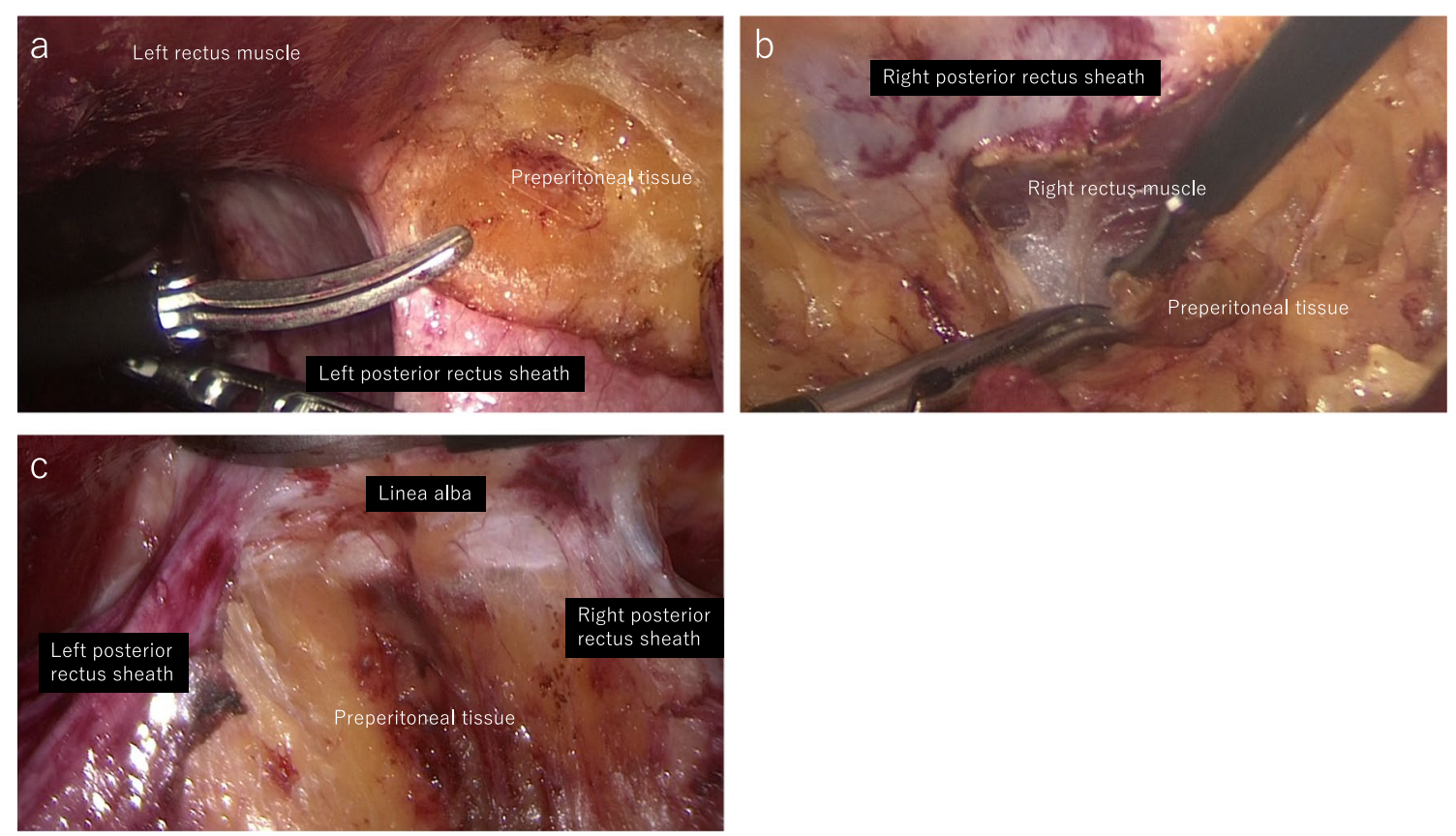

Fig. 3 Intraoperative endoscopic views-1. Intraoperative endoscopic view. a Medial portion of the left posterior rectus sheath was incised. b Medial portion of the right posterior rectus sheath was incised after crossing the preperitoneal space. $\mathbf{c}$ Retrorectus space and preperitoneal space were dissected from the cephalad to caudal direction, incising bilateral posterior rectus sheaths
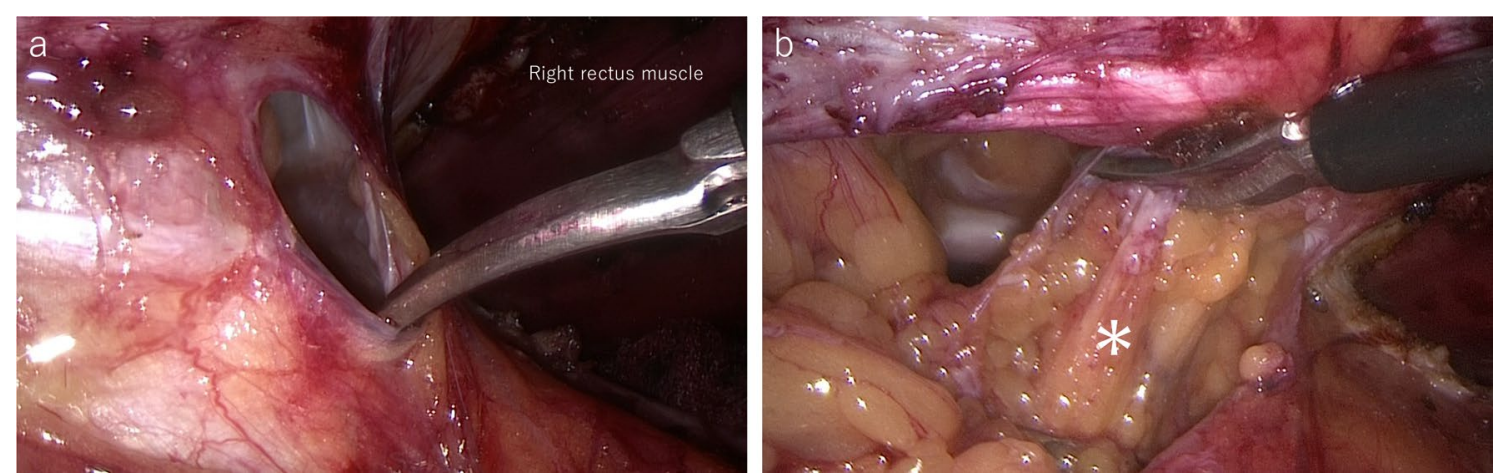

Fig. 4 Intraoperative endoscopic views-2. Intraoperative endoscopic view. a Hernia sac was sharply dissected. b Mild adhesions between the hernia sac and omental tissue $\left(^{*}\right)$ were observed 

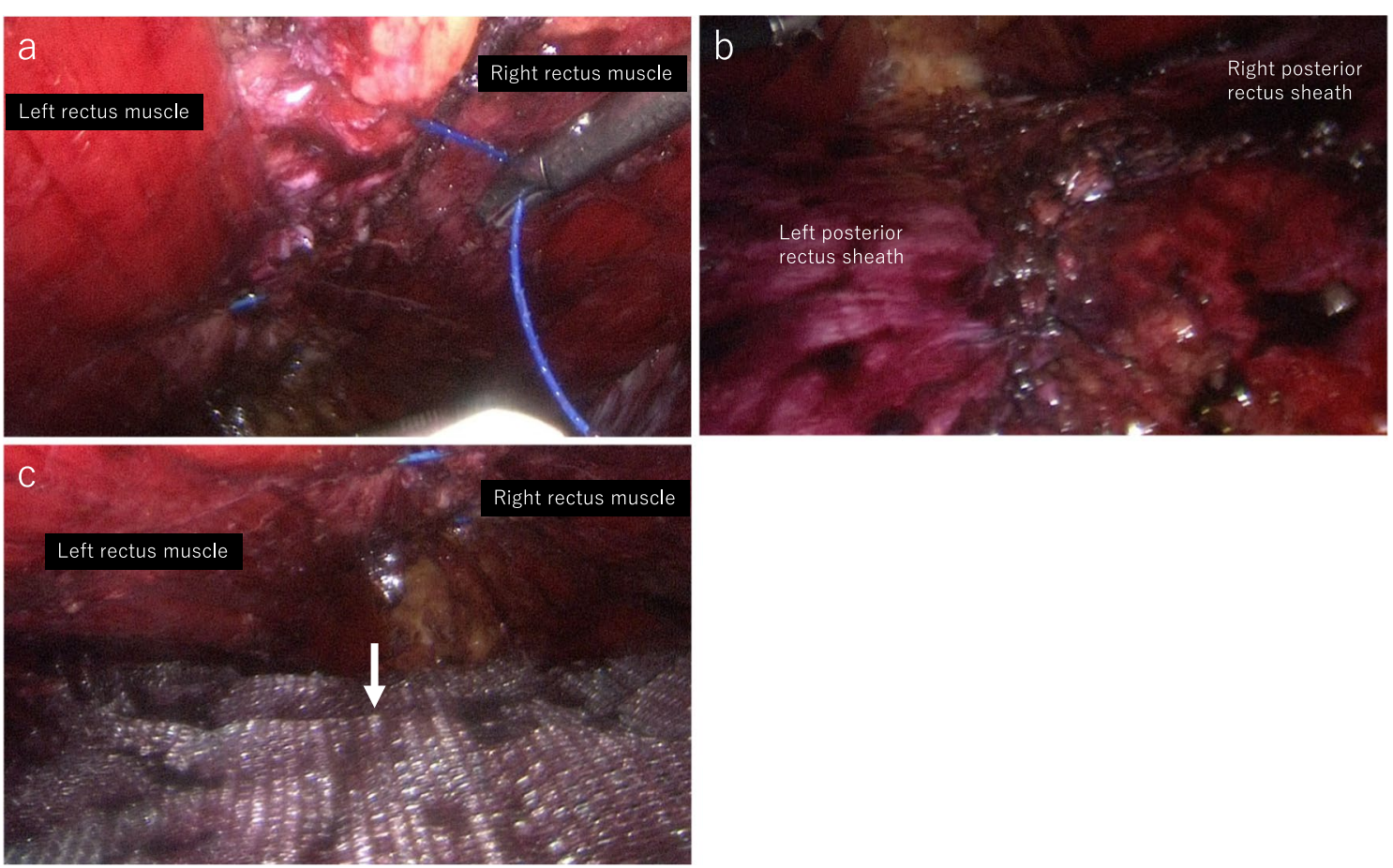

Fig. 5 Intraoperative endoscopic views-3. a Abdominal defect was closed by 0 non-absorbable barbed suture. b Defect of the peritoneum was closed by 2-0 absorbable suture. c 20-cm-long and 15-cm-wide self-gripping mesh was placed in the retrorectus space with no fixation (arrow)
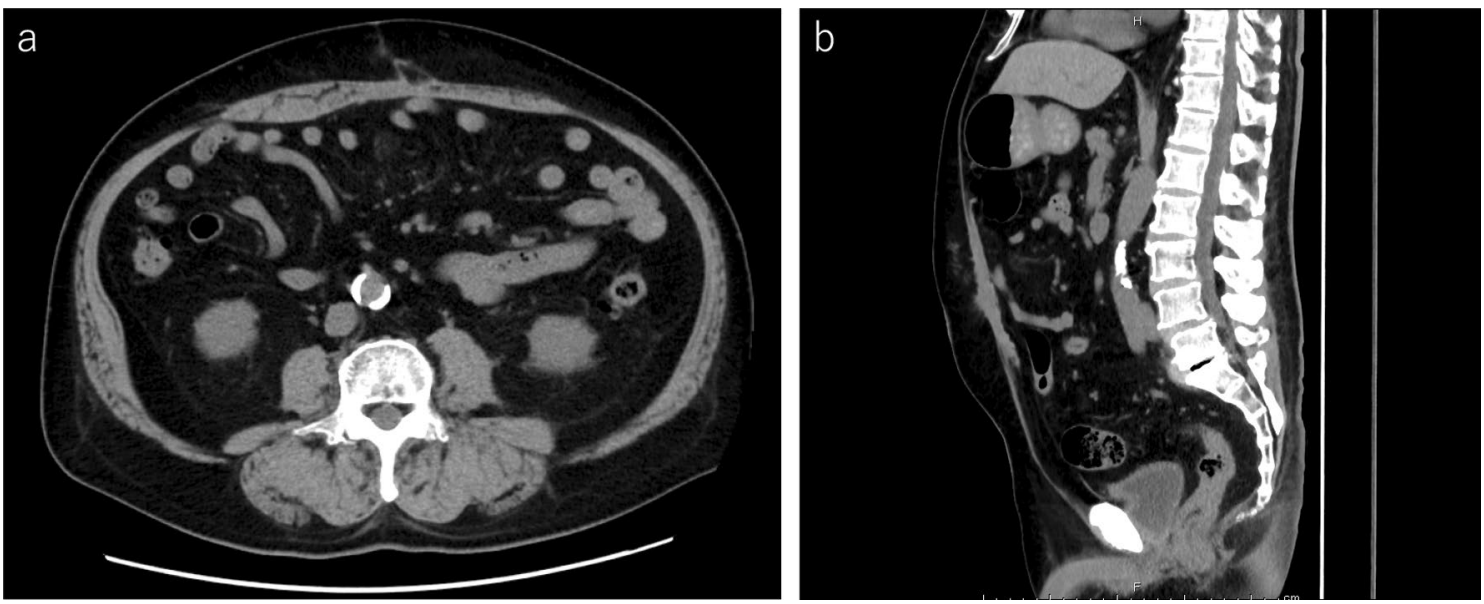

Fig. 6 Post-operative abdominal computed tomography. Abdominal computed tomography at the time of 9 months after surgery shows no hernia recurrences. a Axial image. b Sagittal image

\section{Discussion}

Recent large sample size cohort studies reported that the incisional hernia incidence rate was higher with minimally invasive radical prostatectomy, including RARP, than with traditional open radical prostatectomy $[3,4]$. The incidence rate of incisional hernia following RARP has been reported to be $4.4-8.6 \%$ [8-11]. It is speculated that the incisional hernia occurrence rate after RARP was higher than that after open prostatectomy, because the camera trocar and specimen extraction site were placed above or in the umbilical region in RARP, where the muscles are known to be weaker, as opposed to the infraumbilical incision in open prostatectomy [4]. Given the recent worldwide spread of RARP for the treatment 
of prostate cancer, the number of incisional hernia cases after RARP is expected to increase.

The laparoscopic approach to incisional hernia repair with the IPOM technique was first described by LeBlanc in 1993 [12]. It has been reported that the laparoscopic approach to ventral hernia, including incisional hernia repair, has lower wound complication rates and faster recovery than the open approach [12, 13]. Therefore, laparoscopic repair of ventral hernia is rapidly becoming widespread, especially laparoscopic IPOM, the most popular technique [14]. However, the IPOM technique has serious complications, such as mesh-related adhesive bowel obstruction, mesh erosion, enterocutaneous fistula, and tacker-related chronic pain [15-17]. Postoperative pain has also been reported to be associated with double crown fixation and transfascial sutures [18].

The eTEP technique, which is an endoscopically performed Rives-Stoppa technique, was first described by Miserez for ventral hernia repair [6]. The eTEP can avoid the aforementioned complications by placing a mesh in the retrorectus space, not only eliminating contact between the mesh and intra-abdominal organs, which can avoid mesh-related complications, but also facilitating the minimization of using penetrating fixation, thereby avoiding tacker-related chronic pain as well [7, 19]. In a retrospective comparative analysis, Penchev et al. reported that the differences between eTEP and laparoscopic IPOM for repair of ventral hernias were the reduction in mean postoperative video analog scale pain score and the longer operative time, both in favor of eTEP. They considered that a lack of fixation in eTEP led to a reduction in postoperative pain [19].

When dissecting the hernia sac for eTEP repair, careful procedures are required to avoid injury to intra-abdominal organs, such as the small bowel. We consider that dissecting the hernia sac with a laparoscopic scissors in small steps is useful to avoid damaging the intra-abdominal organs. It is controversial whether a prosthesis can use for the incisional hernia repair if a bowel resection is performed as a result of a bowel injury [20, 21]. Although no studies have been reported on post-RARP adhesions, some studies have reported that laparoscopic surgery reduced adhesion formations for reasons, such as reduced peritoneal incision size, introduction of fewer foreign bodies, and less tissue trauma and bleeding than open surgery $[22,23]$. Therefore, we consider that eTEP repair is one of the best treatment procedures for incisional hernia after minimally invasive surgery. On the other hand, we consider that eTEP repair is challenging for incisional hernia patients with large defects, for postlaparotomy cases, and for recurrence cases. For those challenging incisional hernia cases, endoscopic transversus abdominis muscle release, which is one of the posterior component separation procedures, is useful as a minimally invasive surgery [24]. Belyansky reported that transversus abdominis muscle release was helpful in cases with wide $(>10 \mathrm{~cm})$ defect, tension of the posterior layer, narrow retrorectus space $(<5 \mathrm{~cm})$, or when dealing with a compliant abdominal wall [7].

\section{Conclusion}

eTEP repair is a very useful minimally invasive procedure for patients with incisional hernias after RARP. Although long-term follow-up is necessary to establish the safety and efficacy of eTEP repair, we consider this procedure to be the best option for patients with incisional hernia because of its few postoperative complications.

\section{Abbreviations}

eTEP: Enhanced-view totally extraperitoneal; IPOM: Intraperitoneal onlay mesh; RARP: Robot-assisted laparoscopic radical prostatectomy.

\section{Acknowledgements}

We would like to thank Editage (www.editage.com) for English language editing.

\section{Authors' contributions}

$\mathrm{SH}$ was involved in patient care and wrote the first version of the manuscript. All authors read and approved the final manuscript.

\section{Funding}

This study was not funded.

Availability of data and materials

The data sets supporting the conclusions of this article are included in this paper.

\section{Declarations}

Ethics approval and consent to participate Not applicable.

\section{Consent for publication}

Written informed consent was obtained from the patient for publication of this case report and any accompanying images.

\section{Competing interests}

All of the authors declare no competing interests.

Received: 21 October 2021 Accepted: 31 January 2022

Published online: 07 February 2022

\section{References}

1. Murphy DG, Kerger M, Crowe H, Peters JS, Costello AJ. Operative details and oncological and functional outcome of robotic-assisted laparoscopic radical prostatectomy: 400 cases with a minimum of 12 months followup. Eur Urol. 2009;55(6):1358-66.

2. Du Y, Long Q, Guan B, Mu L, Tian J, Jiang Y, et al. Robot-Assisted radical prostatectomy is more beneficial for prostate cancer patients: a system review and meta-analysis. Med Sci Monit. 2018;24:272-87.

3. Carlsson SV, Ehdaie B, Atoria CL, Elkin EB, Eastham JA. Risk of incisional hernia after minimally invasive and open radical prostatectomy. J Urol. 2013;190(5):1757-62. 
4. Hermann M, Gustafsson O, Sandblom G. Incidence of incisional hernia after minimally invasive and open radical prostatectomy: a populationbased nationwide study. Scand J Urol. 2017:51(4):264-8.

5. Poulose BK, Shelton J, Phillips S, Moore D, Nealon W, Penson D, et al. Epidemiology and cost of ventral hernia repair: making the case for hernia research. Hernia. 2012;16(2):179-83.

6. Miserez M, Penninckx F. Endoscopic totally preperitoneal ventral hernia repair. Surg Endosc. 2002;16(8):1207-13.

7. Belyansky I, Daes J, Radu VG, Balasubramanian R, Reza Zahiri H, Weltz AS, et al. A novel approach using the enhanced-view totally extraperitoneal (eTEP) technique for laparoscopic retromuscular hernia repair. Surg Endosc. 2018:32(3):1525-32.

8. Fuller A, Fernandez A, Pautler SE. Incisional hernia after robot-assisted radical prostatectomy-predisposing factors in a prospective cohort of 250 cases. J Endourol. 2011;25(6):1021-4

9. Beck S, Skarecky D, Osann K, Juarez R, Ahlering TE. Transverse versus vertical camera port incision in robotic radical prostatectomy: effect on incisional hernias and cosmesis. Urology. 2011;78(3):586-90.

10. Chennamsetty A, Hafron J, Edwards L, Pew S, Poushanchi B, Hollander J, et al. Predictors of incisional hernia after robotic assisted radical prostatectomy. Adv Urol. 2015;2015:457305.

11. Timm B, O'Connor E, Bolton D, Liodakis P. Are we failing to consent to an increasingly common complication? Incisional hernias at robotic prostatectomy. J Robot Surg. 2020;14(6):861-4.

12. LeBlanc KA, Booth WV. Laparoscopic repair of incisional abdominal hernias using expanded polytetrafluoroethylene: preliminary findings. Surg Laparosc Endosc. 1993;3(1):39-41.

13. Sauerland S, Walgenbach M, Habermalz B, Seiler CM, Miserez M. Laparoscopic versus open surgical techniques for ventral or incisional hernia repair. Cochrane Database Syst Rev. 2011;3:Cd007781.

14. Bittner JGt, Baghai M, Jacob BP. Management of a primary ventral incisional hernia: a survey of the International Hernia Collaboration. J Robot Surg. 2020;14(1):95-9.

15. LeBlanc KA. Laparoscopic incisional hernia repair: are transfascial sutures necessary? A review of the literature. Surg Endosc. 2007;21(4):508-13.

16. Montgomery A. The best of two worlds: a new innovative laparoscopic Rives-Stoppa technique for ventral/incisional hernias--"the Brazilian technique": Invited commentary to: Transabdominal midline reconstruc tion by minimally invasive surgery: technique and results. Costa TN, Abdalla RZ, Santo MA, Tavares RRFM, Abdalla BMZ, Cecconello I. Hernia. 2016;20(2):267-70.

17. Weltz AS, Sibia US, Zahiri HR, Schoeneborn A, Park A, Belyansky I. Operative outcomes after open abdominal wall reconstruction with retromuscular mesh fixation using fibrin glue versus transfascial sutures. Am Surg. 2017;83(9):937-42.

18. Brill JB, Turner PL. Long-term outcomes with transfascial sutures versus tacks in laparoscopic ventral hernia repair: a review. Am Surg. 2011;77(4):458-65.

19. Penchev D, Kotashev G, Mutafchiyski V. Endoscopic enhanced-view totally extraperitoneal retromuscular approach for ventral hernia repair. Surg Endosc. 2019;33(11):3749-56.

20. Nieuwenhuizen J, van Ramshorst GH, ten Brinke JG, de Wit T, van der Harst E, Hop WC, et al. The use of mesh in acute hernia: frequency and outcome in 99 cases. Hernia. 2011;15(3):297-300.

21. Hentati H, Dougaz W, Dziri C. Mesh repair versus non-mesh repair for strangulated inguinal hernia: systematic review with meta-analysis. World J Surg. 2014;38(11):2784-90.

22. Gutt CN, Oniu T, Schemmer P, Mehrabi A, Büchler MW. Fewer adhesions induced by laparoscopic surgery? Surg Endosc. 2004;18(6):898-906

23. Bartels SA, Vlug MS, Hollmann MW, Dijkgraaf MG, Ubbink DT, Cense $\mathrm{HA}$, et al. Small bowel obstruction, incisional hernia and survival after laparoscopic and open colonic resection (LAFA study). Br J Surg. 2014;101(9):1153-9.

24. Bittner R, Bain K, Bansal VK, Berrevoet F, Bingener-Casey J, Chen D, et al. Update of Guidelines for laparoscopic treatment of ventral and incisional abdominal wall hernias (International Endohernia Society (IEHS)): part B. Surg Endosc. 2019;33(11):3511-49.

\section{Publisher's Note}

Springer Nature remains neutral with regard to jurisdictional claims in published maps and institutional affiliations.

\section{Submit your manuscript to a SpringerOpen ${ }^{\circ}$ journal and benefit from:}

- Convenient online submission

- Rigorous peer review

- Open access: articles freely available online

- High visibility within the field

- Retaining the copyright to your article

Submit your next manuscript at $\boldsymbol{\nabla}$ springeropen.com 